\title{
Long-term monitoring of bioluminescence circadian rhythms of cells in a transgenic Arabidopsis mesophyll protoplast culture
}

\author{
Shunji Nakamura, Tokitaka Oyama* \\ Department of Botany, Graduate School of Science, Kyoto University, Kitashirakawa-oiwake-cho, Sakyo-ku, Kyoto \\ 606-8502, Japan \\ *E-mail: oyama@cosmos.bot.kyoto-u.ac.jp Tel: +81-75-753-4135 Fax: +81-75-753-4137
}

Received March 16, 2018; accepted May 15, 2018 (Edited by R. Hayama)

\begin{abstract}
The circadian system of plants is based on the cell-autonomously oscillating circadian clock. In the plant body, these cellular clocks are associated with each other, but their basic and intrinsic properties are still largely unknown. Here we report a method that enables long-term monitoring of bioluminescence circadian rhythms of a protoplast culture in a complete synthetic medium. From the leaves of Arabidopsis transgenic plants carrying the luciferase gene under a clockgene promoter, mesophyll protoplasts were isolated and their bioluminescence was automatically measured every 20 min for more than one week. Decreasing luminescence intensities were observed in protoplasts when they were cultured in a Murashige and Skoog-based medium and also in W5 solution. This decrease was dramatically improved by adding the phytohormones auxin and cytokinin to the MS-based medium; robust circadian rhythms were successfully monitored. Interestingly, the period lengths of bioluminescence circadian rhythms of protoplasts under constant conditions were larger than those of detached leaves, suggesting that the period lengths of mesophyll cells in leaves were modulated from their intrinsic properties by the influence of other tissues/cells. The entrainability of protoplasts to light/dark signals was clearly demonstrated by using this monitoring system. By analyzing the circadian behavior of isolated protoplasts, the basic circadian system of plant cells may be better understood.
\end{abstract}

Key words: Arabidopsis thaliana, bioluminescence monitoring, circadian rhythm, entrainment, mesophyll protoplasts.

Most organisms possess a circadian rhythm that helps them adapt to environmental day-night cycles. In plants, which depend considerably on sunlight, various physiological phenomena exhibit daily rhythms that are modulated by the circadian clock (Greenham and McClung 2015). Plant circadian clocks are based on transcription-translation feedback loops of a number of clock genes (Nagel and Kay 2012). Arabidopsis CCA1 is one of these clock genes, and its gene expression oscillates with a peak around dawn. It has rhythmic expression with a period of ca. $24 \mathrm{~h}$ under constant conditions. Most clock components encoded by clock genes are transcription factors, and the clock cell-autonomously oscillates (Muranaka and Oyama 2018). Indeed, it was reported that cultured cells of Arabidopsis that were transformed with clockcontrolled luminescence reporter genes showed robust bioluminescence circadian rhythms similar to those of intact plants (Kim et al. 2003; Nakamichi et al. 2004). By measuring rhythmicity in individual leaf cells in transgenic Arabidopsis plants with fluorescence-tagged
CCA1, cell-specific differences in clock properties were demonstrated (Yakir et al. 2011). Furthermore, by developing a single-cell bioluminescence imaging system for monitoring cellular gene expression in duckweed, the circadian behavior of individual cells in an intact plant was quantitatively analyzed (Muranaka and Oyama 2016, 2018; Muranaka et al. 2013; Okada et al. 2017). Individual cellular circadian clocks oscillate with their own frequencies and respond independently to external light/dark signals. Despite the heterogeneous and unstable features of cellular clocks in the duckweed plant, partial synchronization between neighboring cells was suggested. In Arabidopsis as well, cell-to-cell interactions for synchronization of cellular clocks were suggested (Fukuda et al. 2007; Wenden et al. 2012). The circadian rhythms of vascular tissues were also reported to be robust and dominant over those of mesophyll tissues in Arabidopsis (Endo et al. 2014). Studies using Arabidopsis at an organ level suggested that the circadian clock in the shoot apex was robust and dominant over other clocks in leaves and roots (Takahashi et al. 2015).

Abbreviations: CCA1, CIRCADIAN CLOCK ASSOCIATED1; DD, constant dark; FBS, fetal bovine serum; LL, constant light; LUC, luciferase gene; MSP medium, MS-based protoplast medium; PH, phytohormones.

This article can be found at http://www.jspcmb.jp/

Published online July 11, 2018 
Individual circadian clocks in the plant body are thus likely to be influenced in their coordinated behavior by signals of various hierarchies. Information regarding the basic features of cellular circadian rhythms without those influences should be required for better understanding of the plant circadian systems. Protoplasts isolated from plant tissues can be free from contact with other cells in the culture medium; cell-to-cell communications that influence their circadian clocks are thus unlikely. Protoplasts from Arabidopsis leaves transfected with luminescence reporters have been reported to exhibit bioluminescence circadian rhythms (Kim and Somers 2010). This system can be used for a convenient assay of the molecular functions of clock-related genes. Here we report that bioluminescence monitoring of protoplastderived cells of CCA1::LUC transgenic Arabidopsis leaves can be performed long-term by improving the medium conditions. Using this monitoring system, entrainability to light/dark cycles and stable circadian rhythmicity in isolated cells was demonstrated.

We used the previously reported CCA1::LUC transgenic Arabidopsis thaliana (Col-0) (Nakamichi et al. 2004, 2005). Mesophyll protoplasts were isolated from leaves of 3-4-week-old plants aseptically grown in $0.5 \times$ MS (Murashige and Skoog) medium (0.8\% agar) with $1 \%$ sucrose under constant light conditions $\left(32 \mu \mathrm{mol} \mathrm{m}^{-2} \mathrm{~s}^{-1}\right.$ at $22^{\circ} \mathrm{C}$ ) (Figure $\left.1 \mathrm{~A}, \mathrm{~B}\right)$. Before protoplast isolation, these plants were exposed to two 12-h dark periods to entrain (synchronize) circadian rhythms. Protoplast isolation was performed as described by Yoo et al. (2007) with minor modifications. Fifty leaves were detached from plants 3-4h after lights-on (Figure 1A). Enzyme digestion with $20 \mathrm{ml}$ enzyme solution was performed in dim light with gentle shaking for $3 \mathrm{~h}$ at $25^{\circ} \mathrm{C}$. Luciferin was included in the enzyme solution $[400 \mathrm{mM}$ mannitol, $20 \mathrm{mM} \mathrm{KCl}, 20 \mathrm{mM}$ MES-KOH (pH 5.8), $10 \mathrm{mM} \mathrm{CaCl}_{2}$, $1 \%$ Cellulase R10, 0.54\% Macerozyme R10, 0.1\% BSA, $0.1 \mu \mathrm{M}$ luciferin]. The released protoplasts were filtered through a sterile $70 \mu \mathrm{m}$ nylon mesh and harvested by centrifugation at $500 \mathrm{~g}$ for $2 \mathrm{~min}$ at $22^{\circ} \mathrm{C}$. The protoplast pellet was resuspended and washed twice with $45 \mathrm{ml}$ W5 solution $\left[154 \mathrm{mM} \mathrm{NaCl}, 125 \mathrm{mM} \mathrm{CaCl}_{2}, 5 \mathrm{mM} \mathrm{KCl}\right.$, $1.5 \mathrm{mM}$ MES-KOH (pH 5.6)]. The protoplast pellet was then resupended with ca. $4 \mathrm{ml}$ W5 solution. Cells in a $1-\mathrm{ml}$ aliquot were harvested by centrifugation at $100 \mathrm{~g}$ for $3 \mathrm{~min}$ at $22^{\circ} \mathrm{C}$, and the pellet was resuspended with $4 \mathrm{ml}$ culture medium with $0.1 \mu \mathrm{M}$ luciferin. The cell suspension was then transferred into a $35-\mathrm{mm}$ dish for bioluminescence monitoring. Removal of undigested tissues was checked with a microscope. Protoplasts sank to the bottom and they exhibited more than 100 times less bioluminescence density than did leaf cells (Figure 1C). Luminescence intensities of protoplasts changed similarly to the leaf of the CCA1::LUC transgenic plant, implying that the circadian system of
A

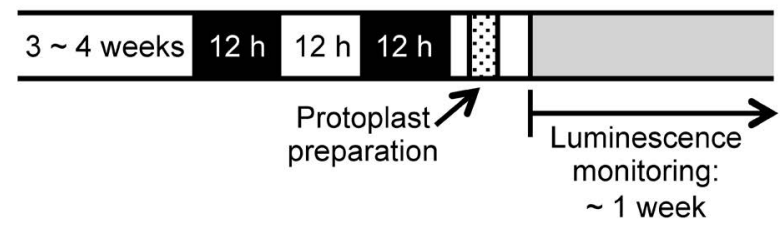

B
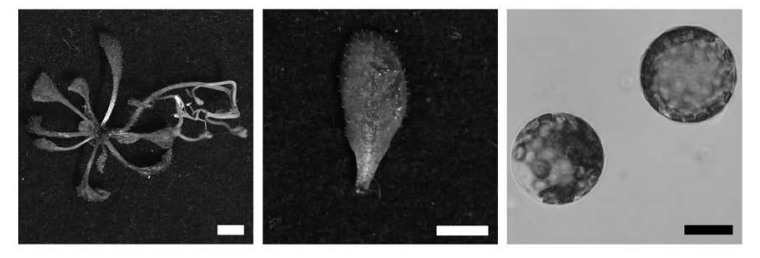

C T 30

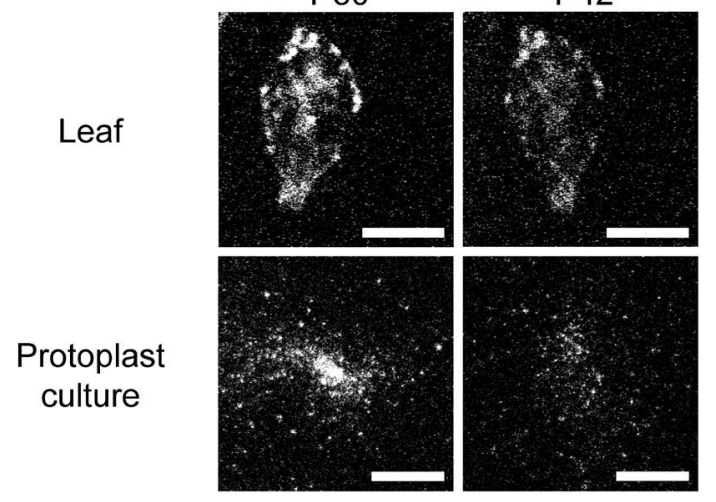

T 42

Figure 1. Protoplasts for monitoring bioluminescence circadian rhythms. (A) Experimental scheme for luminescence monitoring. Black and open bars indicate dark and light, respectively. (B) Protoplasts and their plant materials. Mesophyll protoplasts (right) were isolated from leaves (center) of 4-week-old Arabidopsis plants (left). (C) Luminescence images of a detached leaf (upper) and a protoplast culture (lower). The MSP $+\mathrm{PH}$ medium was used for culturing them. Images were captured $30 \mathrm{~h}$ (left) and $42 \mathrm{~h}$ (right) after the last lights-on by an EM-CCD camera (ImagEM C9100-13; Hamamatsu Photonics) as described previously (Muranaka et al. 2013). The exposure times for leaf and protoplast culture were $1 \mathrm{~s}$ and $240 \mathrm{~s}$, respectively. White scale bars and a black scale bar represent $5 \mathrm{~mm}$ and $20 \mu \mathrm{m}$, respectively.

those protoplasts was robust. To analyze the circadian rhythms of protoplast-derived cells in detail, it was important to monitor their bioluminescence traces for a long duration. The major growth regulators, auxins and cytokinins, are known to be essential for sustained protoplast growth in plants (Davey et al. 2005). We tested an MS-based protoplast medium with or without phytohormones (MSP $+\mathrm{PH}, \mathrm{MSP})$. The MSP medium contains $1 \times \mathrm{MS}$ salts, $1 \%$ sucrose, $400 \mathrm{mM}$ mannitol, $2 \mathrm{mg} / \mathrm{L}$ glycine, $100 \mathrm{mg} / \mathrm{L}$ myo-inositol, $3 \mathrm{mg} / \mathrm{L}$ thiamin hydrochloride, $5 \mathrm{mg} / \mathrm{L}$ nicotinic acid, $0.5 \mathrm{mg} / \mathrm{L}$ pyridoxine hydrochloride, $\mathrm{KOH}$ ( $\mathrm{pH}$ 5.6). Phytohormones $[1 \mu \mathrm{M}$ NAA (1-Naphthaleneacetic Acid), $0.2 \mu \mathrm{M}$ kinetin] were added to the MSP medium to make the MSP $+\mathrm{PH}$ medium (Fukazawa et al. 2014; Satoh et al. 2004).

We first monitored bioluminescence of protoplastderived cells in the three media (MSP $+\mathrm{PH}, \mathrm{MSP}, \mathrm{W} 5)$ 
A

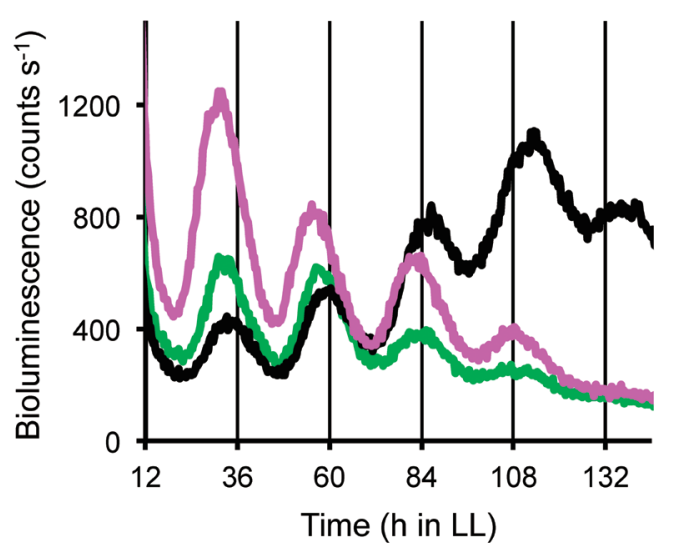

B

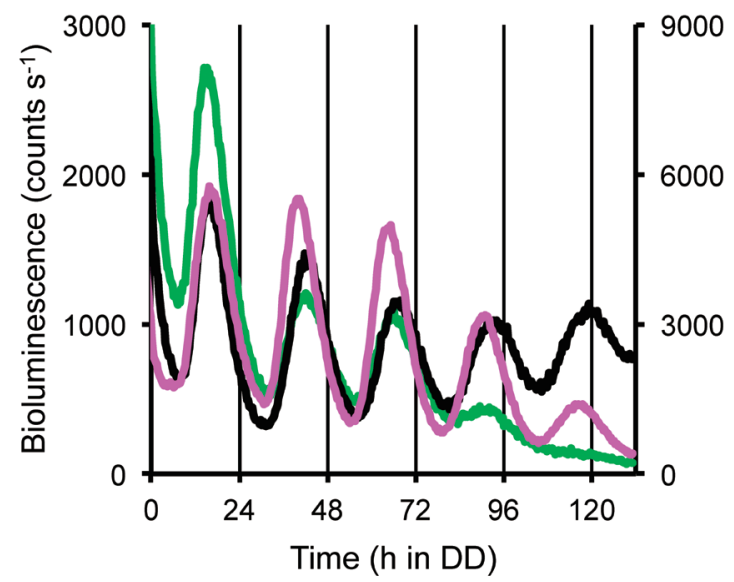

C

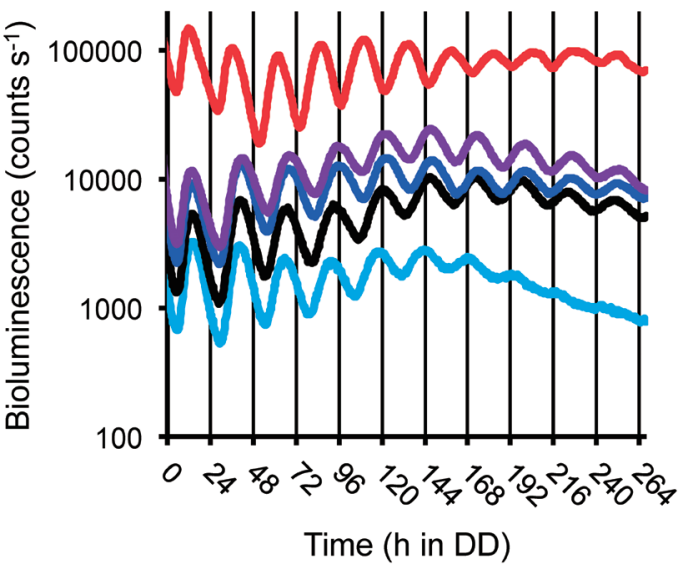

Figure 2. Bioluminescence circadian rhythms of protoplast-derived cells of the CCA1::LUC transgenic Arabidopsis under constant light and constant dark. (A, B) Circadian rhythms of luciferase activities of protoplast-derived cells in MSP $+\mathrm{PH}$ medium (black line), MSP medium (green line), W5 solution (magenta line) under constant light (A) or constant dark (B). Experimental schemes are shown in Figure 1A. Time of panel $\mathrm{A}$ is set as hours in constant light including time before protoplast isolation. Time of panel B is set as hours in constant dark starting $12 \mathrm{~h}$ after the last lights-on. Data for the magenta line in (B) are plotted with scale on right. (C) Long-term monitoring of bioluminescence circadian rhythms under constant dark. Circadian rhythms of protoplast-derived cells $\left[1 \times 10^{5} \mathrm{cells} / 4 \mathrm{ml}\right.$ (light blue line), $2 \times 10^{5} \mathrm{cells} / 4 \mathrm{ml}$ (black line), $4 \times 10^{5}$ cells $/ 4 \mathrm{ml}$ (blue line), $6 \times 10^{5}$ cells $/ 4 \mathrm{ml}$ (purple line)] in MSP $+\mathrm{PH}$ medium and a detached leaf of the CCA1::LUC transgenic Arabidopsis on NF medium (red line). The composition of NF medium was described previously (Muranaka et al. 2015). under constant light. Hereafter, we use "protoplastderived cells" in our experiments for the detection of bioluminescence rhythms because protoplasts tend to begin cell-wall regeneration within $48 \mathrm{~h}$ after protoplast isolation (Sheahan et al. 2004; Tiew et al. 2015). Bioluminescence monitoring was performed as described previously (Muranaka et al. 2015). The bioluminescence dish-monitoring system used photomultiplier tubes for bioluminescence detection, and each dish was subjected to $30 \mathrm{~s}$ measurement every $20 \mathrm{~min}$. Bioluminescence circadian rhythms were observed in all medium conditions under constant light and constant dark (Figure 2A, B). However, luminescence intensities in MSP medium and W5 solution gradually decreased and their rhythms were damped. Under both conditions, the period lengths of bioluminescence rhythms in MSP $+\mathrm{PH}$ were slightly larger than those in MSP medium and W5 solution (Figure 3). The shorter period lengths in these media might be due to the severe damping of rhythms. Meanwhile, the period lengths of protoplast-derived cells in any media were larger than those of detached leaves, especially under constant light (Figure 3). Since sustainability in luminescence intensities and its rhythms of protoplast-derived cells in $\mathrm{MSP}+\mathrm{PH}$ was shown, we next examined the rhythmicity under prolonged constant dark conditions. We tested protoplast-derived cells at various densities $\left(1 \times 10^{5}-6 \times 10^{5}\right.$ cells in $4 \mathrm{ml}$ $\mathrm{MSP}+\mathrm{PH}$ medium). At all densities, luminescence intensities were maintained for 11 days with gradual increase for ca. 5 days and gradual decrease afterwards (Figure 2C). At the three highest cell densities $\left(2 \times 10^{5}\right.$ $6 \times 10^{5}$ cells), the circadian rhythms were maintained for

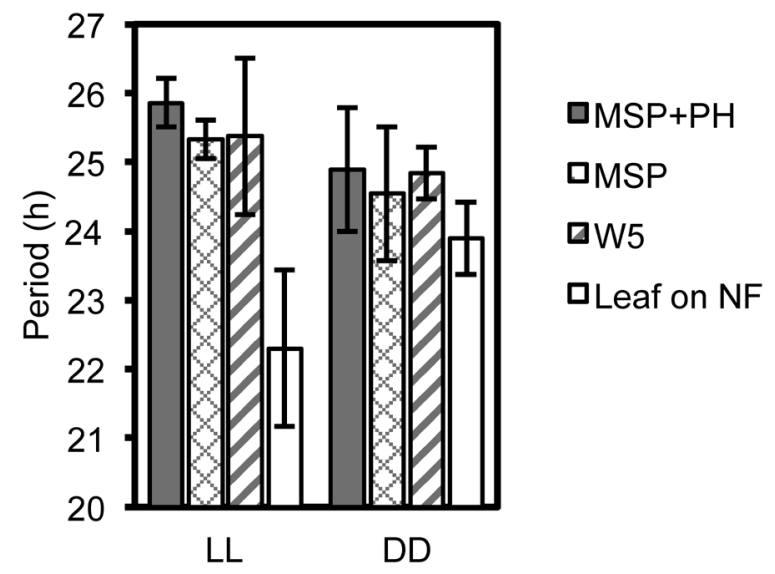

Figure 3. Period lengths of bioluminescence circadian rhythms under constant light and constant dark in various medium conditions. Circadian rhythms of protoplast-derived cells in MSP $+\mathrm{PH}$ medium ( $n=9$ in LL ; $n=15$ in DD), MSP medium ( $n=9$ in LL; $n=18$ in DD), W5 solution ( $n=9$ in LL; $n=15$ in DD), and leaves on NF ( $n=12$ in LL; $n=12$ in DD) were monitored as described in Figure 2. The period of each rhythm was estimated by FFT-NLLS, a multicomponent cosine fit (Zielinski et al. 2014). Luminescence data in the range between $24 \mathrm{~h}$ and $96 \mathrm{~h}$ in constant conditions were used for the analysis. Mean values with error bars (SD) are represented in the bar graph. 
11 days with gradual damping that was also observed in the bioluminescence rhythm of a detached leaf. At the lowest cell density $\left(1 \times 10^{5}\right.$ cells $)$, the circadian rhythm was maintained for 6 days with gradual damping and then showed severe damping, suggesting that higher cell densities were required for long-term monitoring of bioluminescence rhythms of protoplast-derived cells.

Protoplast-derived cells were only analyzed for their circadian rhythms under free-running conditions in previous studies (Hansen and Ooijen 2016; Kim and Somers 2010; Takahashi et al. 2015; Wang et al. 2013). We then tested the entrainability of the circadian rhythm in protoplast-derived cells. After protoplast isolation, cells were treated with two 12-h dark periods in phase with the light/dark cycles before protoplast isolation (Figure 4A, filled circles). The first peak occurred $2.4 \mathrm{~h}$ after lights-on and the next one occurred at $27.7 \mathrm{~h}$ under constant light. Those cells that were treated with a 12-h dark period in antiphase with the light/dark cycles showed a bioluminescence rhythm (open circles) in antiphase with that of the filled circles (Figure 4A). This indicated that individual protoplast-derived cells were capable of entraining their circadian rhythms to the 12 -h dark period through light/dark responses. Without the entrainment of the dark period, the amplitude of the bioluminescence rhythm was lower than that in entrained cells, suggesting that the circadian rhythms of individual protoplasts became synchronous due to the dark entrainment (Figure 4A, crosses). Detached leaves that tended to show damped rhythms under constant light were entrained to the 12 -h dark period similarly to protoplast-derived cells, suggesting that entrainment behaviors in the dark treatment were similar between them (Figure 4B). This also implied that mesophyll cells in leaves might cell-autonomously respond to the light/ dark signals to entrain their circadian rhythms.

In this study, we successfully monitored the bioluminescence rhythms of protoplast-derived cells of CCA1::LUC transgenic Arabidopsis leaves. Bioluminescence rhythms of those cells were sustained for more than 10 days by improving medium conditions. The monitoring of bioluminescence rhythms of Arabidopsis protoplasts was previously reported by Kim and Somers (2010). A circadian reporter gene was transiently transfected into mesophyll protoplasts and bioluminescence of those cells in W5 solution supplemented with $5 \%$ fetal bovine serum (FBS) was monitored under constant conditions. Adding serum into W5 solution was shown to be effective in the prolonged measurement of protoplast bioluminescence though the mechanism of action was unknown. In our monitoring procedure, by using protoplasts of Arabidopsis transformants, bioluminescence monitoring is begun just after the protoplast isolation. The quick and simple steps for preparation of luminescent protoplasts
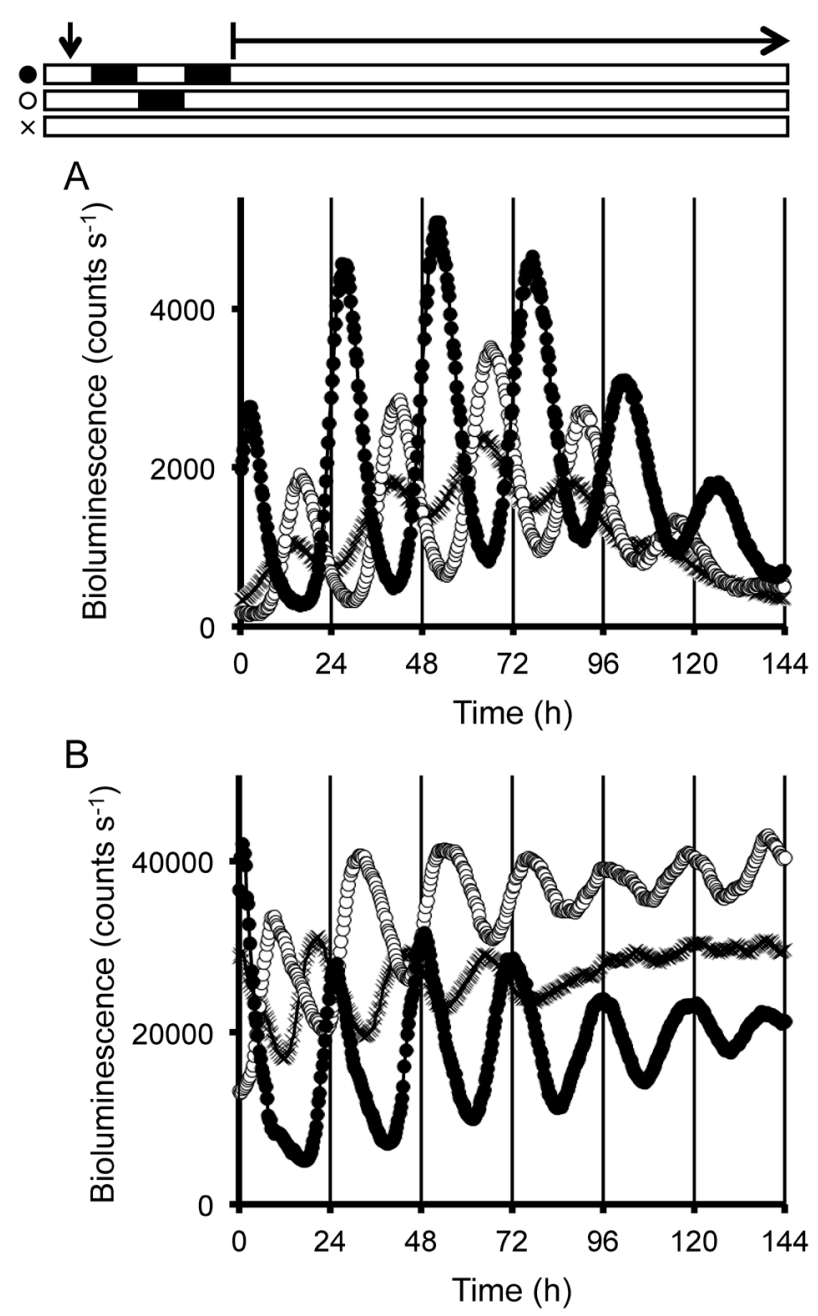

Figure 4. Entrainability of circadian rhythms to light/dark signals. Bioluminescence traces of CCA1::LUC activities of cells in MSP $+\mathrm{PH}$ medium (A) and leaves on NF medium (B) under constant light are plotted. The light/dark schedule for each sample is indicated at the top of graph with its index (filled circle, open circle, cross). A 12-h dark period is indicated as a black box, and the timing of protoplast isolation (A) or leaf detachment (B) is indicated by an arrow. Before protoplast isolation and leaf detachment, plants were treated with two 12-h dark periods as shown in Figure 1A.

is likely to guarantee the uniformity and intactness of cells that is required for reproducible results in longterm monitoring. Adding an auxin and a cytokinin into MS-based medium is critical for the prolonged measurement of protoplast bioluminescence. These phytohormones may partially induce dedifferentiation of protoplast-derived cells and lead to the survival of these cells for a long time. The MS-based medium containing the phytohormones is a complete synthetic medium, whereas the W5 solution supplemented with $5 \% \mathrm{FBS}$ is a semisynthetic medium that enables longterm bioluminescence monitoring of protoplast-derived cells. The complete synthetic medium can be used for experiments to assess chemicals that affect cellular circadian rhythms. Circadian behavior of protoplastderived cells in any culture media showed a tendency 
for period lengths larger than those of detached leaves (Figure 3). This suggested that period lengths of individual mesophyll cells were modulated by other tissues and/or each other in leaves, and protoplastderived cells exhibited the intrinsic period lengths. By analyzing the circadian behavior of isolated protoplastderived cells in a culture medium, the basic circadian system, without influence from other tissues/cells, may be understood.

\section{Acknowledgements}

We thank Dr. Norihito Nakamichi for experimental materials and Drs. Yousuke Takahashi and Jutarou Fukazawa for fruitful discussions. We also thank Drs. Shogo Ito and Masaaki Okada for experimental support. This work was supported in part by the Japan Society for the Promotion of Science KAKENHI (Grant numbers 23657033, 25650098, and 17KT0022), the Iwatani Naoji Foundation, and the Japan Science and Technology Agency (JST) ALCA to T. O.

\section{References}

Davey MR, Anthony P, Power JB, Lowe KC (2005) Plant protoplasts: Status and biotechnological perspectives. Biotechnol Adv 23: 131-171

Endo M, Shimizu H, Nohales MA, Araki T, Kay SA (2014) Tissuespecific clocks in Arabidopsis show asymmetric coupling. Nature 515: 419-422

Fukazawa J, Teramura H, Murakoshi S, Nasuno K, Nishida N, Ito T, Yoshida M, Kamiya Y, Yamaguchi S, Takahashi Y (2014) DELLAs function as coactivators of GAI-ASSOCIATED FACTOR1 in regulation of gibberellin homeostasis and signaling in Arabidopsis. Plant Cell 26: 2920-2938

Fukuda H, Nakamichi N, Hisatsune M, Murase H, Mizuno T (2007) Synchronization of plant circadian oscillators with a phase delay effect of the vein network. Phys Rev Lett 99: 098102

Greenham K, McClung CR (2015) Integrating circadian dynamics with physiological processes in plants. Nat Rev Genet 16: 598-610

Hansen LL, van Ooijen G (2016) Rapid analysis of circadian phenotypes in Arabidopsis protoplasts transfected with a luminescent clock reporter. J Vis Exp 115: e54586

Kim J, Somers DE (2010) Rapid assessment of gene function in the circadian clock using artificial microRNA in Arabidopsis mesophyll protoplasts. Plant Physiol 154: 611-621

Kim WY, Geng R, Somers DE (2003) Circadian phase-specific degradation of the F-box protein ZTL is mediated by the proteasome. Proc Natl Acad Sci USA 100: 4933-4938

Muranaka T, Kubota S, Oyama T (2013) A single-cell bioluminescence imaging system for monitoring cellular gene expression in a plant body. Plant Cell Physiol 54: 2085-2093

Muranaka T, Okada M, Yomo J, Kubota S, Oyama T (2015)
Characterisation of circadian rhythms of various duckweeds. Plant Biol 17(Suppl 1): 66-74

Muranaka T, Oyama T (2016) Heterogeneity of cellular circadian clocks in intact plants and its correction under light-dark cycles. Sci Adv 2: e1600500

Muranaka T, Oyama T (2018) Monitoring circadian rhythms of individual cells in plants. J Plant Res 131: 15-21

Nagel DH, Kay SA (2012) Complexity in the wiring and regulation of plant circadian networks. Curr Biol 22: R648-R657

Nakamichi N, Ito S, Oyama T, Yamashino T, Kondo T, Mizuno T (2004) Characterization of plant circadian rhythms by employing Arabidopsis cultured cells with bioluminescence reporters. Plant Cell Physiol 45: 57-67

Nakamichi N, Kita M, Ito S, Yamashino T, Mizuno T (2005) PSEUDO-RESPONSE REGULATORS, PRR9, PRR7 and PRR5, together play essential roles close to the circadian clock of Arabidopsis thaliana. Plant Cell Physiol 46: 686-698

Okada M, Muranaka T, Ito S, Oyama T (2017) Synchrony of plant cellular circadian clocks with heterogeneous properties under light/dark cycles. Sci Rep 7: 317

Satoh R, Fujita Y, Nakashima K, Shinozaki K, YamaguchiShinozaki K (2004) A novel subgroup of bZIP proteins functions as transcriptional activators in hypoosmolarity-responsive expression of the ProDH gene in Arabidopsis. Plant Cell Physiol 45: 309-317

Sheahan MB, Rose RJ, McCurdy DW (2004) Organelle inheritance in plant cell division: the actin cytoskeleton is required for unbiased inheritance of chloroplasts, mitochondria and endoplasmic reticulum in dividing protoplasts. Plant $J 37$ : 379-390

Takahashi N, Hirata Y, Aihara K, Mas P (2015) A hierarchical multi-oscillator network orchestrates the Arabidopsis circadian system. Cell 163: 148-159

Tiew TW, Sheahan MB, Rose RJ (2015) Peroxisomes contribute to reactive oxygen species homeostasis and cell division induction in Arabidopsis protoplasts. Front Plant Sci 6: 658

Wang L, Kim J, Somers DE (2013) Transcriptional corepressor TOPLESS complexes with pseudoresponse regulator proteins and histone deacetylases to regulate circadian transcription. Proc Natl Acad Sci USA 110: 761-766

Wenden B, Toner DL, Hodge SK, Grima R, Millar AJ (2012) Spontaneous spatiotemporal waves of gene expression from biological clocks in the leaf. Proc Natl Acad Sci USA 109: 6757-6762

Yakir E, Hassidim M, Melamed-Book N, Hilman D, Kron I, Green RM (2011) Cell autonomous and cell-type specific circadian rhythms in Arabidopsis. Plant J 68: 520-531

Yoo S, Cho Y, Sheen J (2007) Arabidopsis mesophyll protoplasts: A versatile cell system for transient gene expression analysis. Nat Protoc 2: 1565-1572

Zielinski T, Moore AM, Troup E, Halliday KJ, Millar AJ (2014) Strengths and limitations of period estimation methods for circadian data. PLoS One 9: e96462 